\title{
TGF $\beta$ signaling in the brain increases with aging and signals to astrocytes and innate immune cells in the weeks after stroke
}

\author{
Kristian P Doyle, Egle Cekanaviciute, Lauren E Mamer, Marion S Buckwalter
}

\begin{abstract}
Background: TGF $\beta$ is both neuroprotective and a key immune system modulator and is likely to be an important target for future stroke therapy. The precise function of increased TGF- $\beta 1$ after stroke is unknown and its pleiotropic nature means that it may convey a neuroprotective signal, orchestrate glial scarring or function as an important immune system regulator. We therefore investigated the time course and cell-specificity of TGF $\beta$ signaling after stroke, and whether its signaling pattern is altered by gender and aging.

Methods: We performed distal middle cerebral artery occlusion strokes on 5 and 18 month old TGF $\beta$ reporter mice to get a readout of TGF $\beta$ responses after stroke in real time. To determine which cell type is the source of increased TGF $\beta$ production after stroke, brain sections were stained with an anti-TGF $\beta$ antibody, colocalized with markers for reactive astrocytes, neurons, and activated microglia. To determine which cells are responding to TGF $\beta$ after stroke, brain sections were double-labelled with anti-pSmad2, a marker of TGF $\beta$ signaling, and markers of neurons, oligodendrocytes, endothelial cells, astrocytes and microglia.

Results: TGF $\beta$ signaling increased 2 fold after stroke, beginning on day 1 and peaking on day 7 . This pattern of increase was preserved in old animals and absolute TGF $\beta$ signaling in the brain increased with age. Activated microglia and macrophages were the predominant source of increased TGF $\beta$ after stroke and astrocytes and activated microglia and macrophages demonstrated dramatic upregulation of TGF $\beta$ signaling after stroke. TGF $\beta$ signaling in neurons and oligodendrocytes did not undergo marked changes.
\end{abstract}

Conclusions: We found that TGF $\beta$ signaling increases with age and that astrocytes and activated microglia and macrophages are the main cell types that undergo increased TGF $\beta$ signaling in response to post-stroke increases in TGF $\beta$. Therefore increased TGF $\beta$ after stroke likely regulates glial scar formation and the immune response to stroke.

\section{Background}

Transforming Growth Factor $\beta 1$ (TGF- $\beta 1$ ) is universally induced by acute and chronic brain injury, including stroke, trauma, seizure, multiple sclerosis, and Alzheimer's disease [1]. TGF $\beta$ is highly conserved and in mammals exists as three isoforms that bind to the same receptors; TGF- $\beta 1$, TGF- $\beta 2$ and TGF- $\beta 3$. TGF- $\beta 1$ is the isoform typically induced by injury [2]. In the brain, TGF $\beta$ receptors are present on all major cell types [3]. However, both TGF $\beta$ activation and signaling are

\footnotetext{
* Correspondence: marionb@stanford.edu

Departments of Neurology and Neurological Sciences, and Neurosurgery, Stanford University Medical School, Stanford, CA, 94305-5489, USA
}

extensively regulated and due to this its effects are typically pleiotrophic and context-dependent. Therefore, after brain injury the biological effects of TGF $\beta$ signaling are likely influenced by both injury type and timing.

After brain injury, TGF $\beta$ signaling can be neuroprotective, but also promote glial scarring and fibrosis [1,4-6]. While neuroprotection is undoubtedly desirable, glial scarring and fibrosis are less likely to be advantageous. In addition, TGF $\beta$ 's have potent effects on the immune system. TGF- $\beta 1$ can be stimulatory or inhibitory depending on the cell type, cytokine milieu and differentiation state of the responding cell and can have both pro- and anti-inflammatory effects [7]. It is not known which subset of these roles TGF- $\beta 1$ plays when 
it is induced by brain injury and how this varies in different types of brain injury.

In stroke, TGF- $\beta 1$ mRNA is elevated for at least a week afterwards [8] and clearly exerts a neuroprotective role. When overexpressed, TGF- $\beta 1$ limits stroke size $[4,9,10]$. And when TGF $\beta$ signaling is blocked, ischemic damage is exacerbated [11]. Because of this, it may be an effective therapeutic agent for stroke. Developing new therapeutic agents to treat those affected by stroke is critical. The elderly segment of the American population is rapidly increasing and $70 \%$ of the deaths of individuals over 65 are attributable to cardiovascular disease [12]. But in order to develop TGF $\beta$ as a therapeutic agent, it is necessary to understand if it plays any additional roles in the brain after stroke.

We therefore investigated the timecourse and cellspecificity of TGF $\beta$ signaling after stroke, and whether its signaling pattern is altered by gender and aging in a mouse model of stroke. We report here that TGF $\beta$ signaling increases 2 fold after the dMCAO model of stroke, beginning on day 1 and peaking on day 7 . This pattern of increase is similar in each gender and preserved in old animals, although absolute TGF $\beta$ signaling was much higher in the brains of the aged animals. CD68+ activated microglia and macrophages were the predominant source of increased TGF- $\beta 1$ after stroke and astrocytes and CD68+ cells were the main cell types that responded to the post-stroke increase in TGF- $\beta 1$. Our results demonstrate that TGF $\beta$ signaling peaks at 7 days after $\mathrm{dMCAO}$ stroke, increases with aging, and that the cells with increased responses during this time period, astrocytes and microglia, are those involved in glial scarring and innate immune responses. This may indicate that TGF $\beta$ signaling plays a role in regulating both the immune response and the glial scar after stroke.

\section{Methods}

\section{Animals}

SBE-LucRT mice were a gift from Dr Tony Wyss-Coray [13]. Nine females and 5 males (5 months old) were used to gauge variability in bioluminescence between individual mice, and to determine if bioluminescence is affected by the estrus cycle. Eighteen young females ( 5 months old) and eighteen old females (18 months old) were used to measure TGF $\beta$ signaling after stroke in real time by bioluminescence. 3 mice from each cohort were sacrificed at days $1,3,7,14$ and 21 after stroke for localization of TGF- $\beta 1$ and TGF $\beta$ signaling by immunoflorescence. Three mice of each age group also underwent sham surgery and were imaged for 21 days prior to sacrifice for localization of TGF $\beta$ signaling in uninjured brains. This experimental paradigm was repeated in young and old male mice.

\section{Surgery}

Distal middle cerebral artery occlusion (dMCAO) was induced as described previously [14]. Briefly, mice were anesthetized by isoflurane inhalation (2\% isoflurane in $100 \%$ oxygen) and the skin over the right temple was shaved. Skin was swabbed with chlorehexidine and an incision was made to expose the temporalis muscle. A pocket was created in the temporalis muscle to expose the skull underneath and the right middle cerebral artery (MCA) was identified. A microdrill was used to penetrate the skull and expose the underlying MCA. The meninges were cut and the vessel was ligated using a small vessel cauterizer. The temporalis muscle was replaced and the wound closed using surgical glue. Throughout surgery body temperature was maintained at $37^{\circ} \mathrm{C}$ using a feedback controlled heating blanket.

\section{Bioluminescent imaging}

Bioluminescence was detected as described previously using the In Vivo Imaging System (IVIS; Xenogen, Alameda, CA) [13]. Mice were injected intraperitoneally with $150 \mathrm{mg} / \mathrm{kg}$ D-luciferin (Xenogen) $10 \mathrm{~min}$ before imaging and anesthetized with isoflurane during imaging. Photons emitted from living mice were acquired as photons per second per $\mathrm{cm}^{2}$ per steradian (sr) by using LIVINGIMAGE software (Xenogen) and integrated over $5 \mathrm{~min}$. For photon quantification, a region of interest was manually selected and kept constant within all experiments. Baseline imaging was performed 1 week before stroke was induced and bioluminescence was expressed as fold induction over baseline levels.

\section{Estrus cycle determination}

For determination of stage of estrus, sterile cotton swabs moistened with saline were inserted into the vagina and gently rotated. Swabs were then rolled onto glass slides and allowed to dry. Polymorphonuclear lymphocytes (PMNs) and epithelial cells were visualized with a Hema 3 Stat Pack (Fisher Cat\#23-123-869) used according to the manufactuer's directions. Stage of estrus cycle was determined based on the ratio of PMN's to nucleated and cornified epithlieal cells. Diestrus was defined as the stage when cells consisted mainly of polymorphonuclear lymphocytes (PMNs). Proestrus was defined as the stage when nucleated and cornified epithelial cells were observed, along with a few PMNs. Estrus was classified as the stage when cornified epithelial cells were predominant, and metestrus was defined as the stage when cornified epithelial cells and PMNs were detected.

\section{Infarct evaluation}

To visualize the region of infarction, representative animals from each age group $(n \geq 5)$ were sacrificed at 24 hours post stroke and their brains processed for TTC 
staining. Brains were sectioned into $1 \mathrm{~mm}$ thick slices and each slice was immersed in 1.5\% TTC in PBS at $37^{\circ} \mathrm{C}$ for 15 minutes and then fixed in $10 \%$ formalin. The area of the ipsilateral hemisphere and the area of the infarct on each section was measured in a blinded fashion using NIH ImageJ 1.43u. The measurements were multiplied by the distance between sections ( 1 $\mathrm{mm}$ ) and then summed over the entire brain to yield volume measurements.

\section{Immunoflorescence}

Immunoflorescence was performed on PFA fixed freefloating coronal brain sections $(40 \mu \mathrm{m})$ using standard techniques. The following primary antibodies were used: anti-TGF- $\beta 1$ (1:1000; Torrey Pines Biolabs, East Orange, NJ), anti-pSmad2 (1:1000; Cat\# AB3849, Millipore, Billerica, MA); Milli-Mark Pan Neuronal marker (1:500; Millipore, Billerica, MA); anti-GFAP (1:1000; Dako, Carpinteria, CA); anti-CD68 (1:1000; ABD Serotec, Raleigh, NC); anti CAII (1:2000; ABD Serotec, Raleigh, NC) and anti- $\beta$-dystroglycan (1:1000; Novacastra/Leica Microsystems, Bannockburn, IL). For quantification of MilliMark Pan Neuronal marker, CAII, CD68 and GFAP colocalization with pSmad2, two sections from each mouse spaced $300 \mu \mathrm{m}$ apart were immunostained. From each section four random fields of view in the penumbra (40x objective) were analyzed. The total number of cells positive for each cell marker was averaged and the percentage of each population that was also positive for pSmad2 was calculated.

\section{Western blotting}

Unstroked and stroked brains were lysed in glo lysis buffer (Promega). Protein concentrations were equalized and lysates mixed with $4 \times$ NuPage LDS loading buffer (Invitrogen). Samples were loaded onto 4-12\% NuPage Bis-Tris gels (Invitrogen) and subsequently transferred onto PVDF membranes. Blots were incubated with rabbit polyclonal antibodies (1:1000) against pSmad2 (cat\# AB3849 from Millipore and cat\# 3101 from Cell Signaling) and actin (cat\# A5060 Sigma-Aldritch) and horseradish peroxidase-conjugated goat anti-rabbit IgG as secondary antibody. Protein signals were detected using an ECL kit (Amersham Pharmacia Biotech) and band intensities were quantified on the original digital image using UN-Scan-It Gel 6.1.

\section{Statistical analysis}

Data was acquired in a blinded and unbiased fashion. Data are expressed as mean $+/$ - standard error of the mean (SEM). Statistical analyses were performed with Prism 5 software (GraphPad, San Diego, CA). Means between two groups were compared with two-tailed, unpaired Student's $t$ tests; comparisons of means from multiple groups with one control were analyzed with one-way ANOVA and Dunnet's post hoc test.

\section{Results}

TGF $\beta$ signaling in the brain is equivalent in males and females and does not fluctuate with the estrus cycle

TGF $\beta$ pathway activation is transmitted primarily through the Smad system. Receptor-regulated Smads (Smad 1, 2, 3, 5 or 8) are phosphorylated, associate with a co-Smad (Smad 4) and translocate to the nucleus, where they bind to Smad binding elements (SBE) to activate transcription of target genes $[15,16]$. SBELucRT mice are TGF $\beta$ reporter mice that express a fusion protein consisting of luciferase (luc), red fluorescent protein $(\mathrm{R})$ and thymidine kinase $(\mathrm{T})$ under the control of a SBE [13]. In these mice luciferase bioluminescence is predominantly in the brain, reliably indicates TGF $\beta$ signaling, and can be followed in real time $[13,17]$. To determine how comparable reporter gene expression is between individual SBE-LucRT mice, and if reporter gene expression fluctuates with gender and the estrus cycle, we imaged nine female and 5 male SBE-LucRT mice for ten days. To determine which stage of the estrus cycle each female was in, a vaginal smear was taken every day and stained with a modified Wright-Giemsa stain. The number of polymorphonuclear leukocytes (PMNs) present in each smear was compared to the number of nucleated and cornifed epithelial cells (Fig 1A). There was no significant difference in bioluminescence between each female or for each stage of the estrus cycle (Fig 1B-D). We also found reporter gene expression to be comparable between male mice and equivalent to that of the females (Fig 1E). This indicates that bioluminescence in SBE-LucRT mice is very comparable between individuals and that TGF $\beta$ signaling in the brain does not fluctuate with gender or the estrus cycle.

\section{TGF $\beta$ signaling increases with age and stroke}

The AMCAO model of stroke produces a small lesion in the cortex with a clearly defined border and a small penumbra. In line with previous reports infarct volume was significantly larger in the aged mice and astrogliosis (GFAP immunoreactivity) was accelerated (Fig 2A, B) $[18,19]$. Bioluminescence in the 5 month old females was increased 2 fold on day 1 post stroke, remained elevated for 7 days post stroke and was localized to the region of the infarct (Fig 2C). After 7 days TGF $\beta$ signaling began to return to baseline (Fig 2D). This fold increase was similar in the 18 month old female mice (Fig 2E), although the absolute amount of TGF $\beta$ signaling in the old mice was substantially higher (Fig 2F). This is not surprising considering that the lesion was much larger in the aged animals. However, TGF $\beta$ 


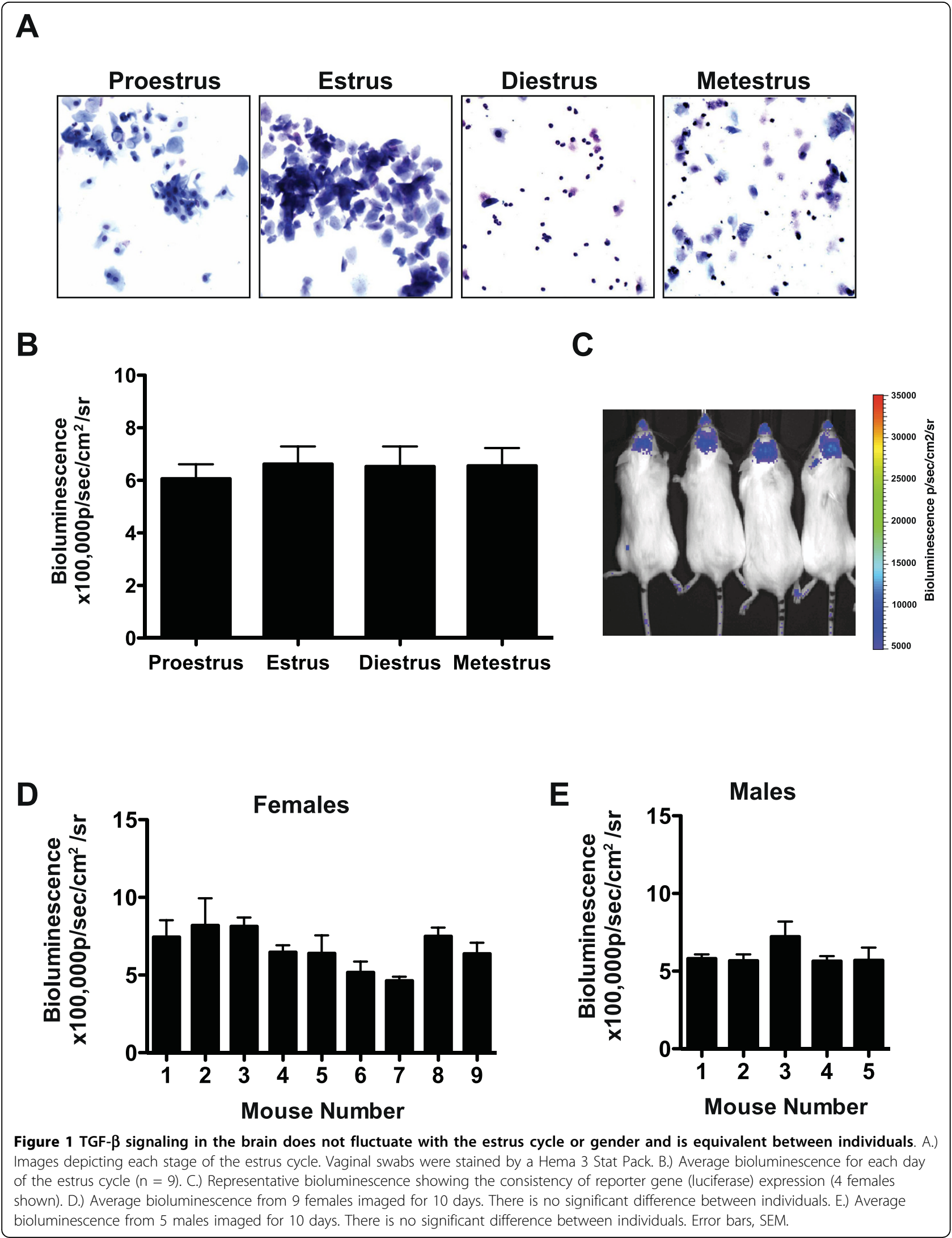




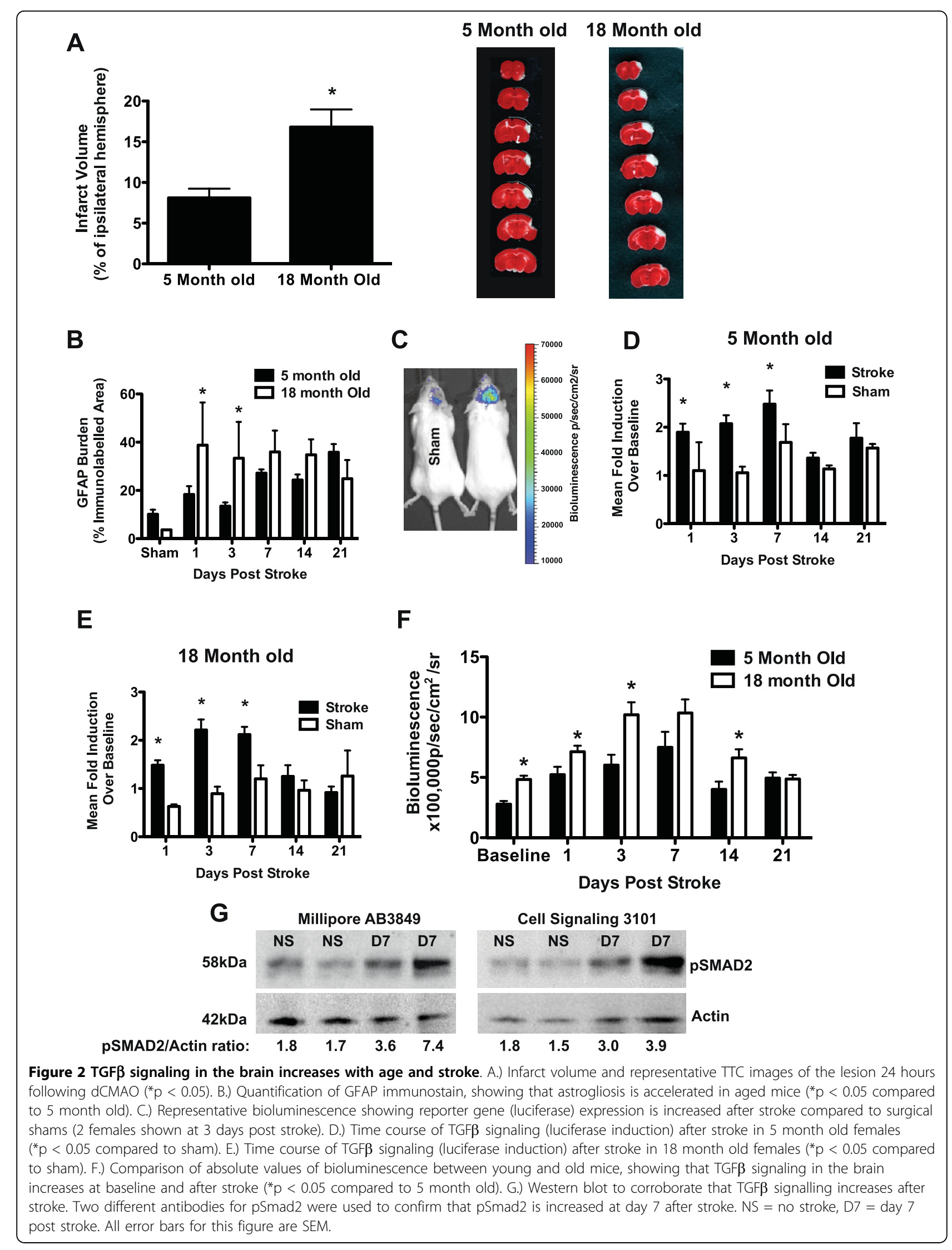


signaling was also significantly higher in the old animals at baseline, demonstrating that in the absence of injury TGF $\beta$ signaling in the brain increases with age.

To confirm the increase in TGF $\beta$ signaling we detected by luciferase activity, we performed a western blot on brain extracts from the ipsilateral hemisphere of mice sacrificed before, and 7 days after stroke, for phosphorylated Smad2 (pSmad2), a downstream mediator of TGF $\beta$ signaling. The pSmad2 antibody we used for immunoflorescence (Millipore AB3849) detected a protein with the correct molecular weight for pSmad2 (58 $\mathrm{kDa}$ ) and bound the same band as an alternative pSmad2 antibody (Cell Signaling 3101; Fig 2G). This verifies that the pSmad2 antibody we used for subsequent immunoflorescence is specific for $\mathrm{pSmad} 2$. In accordance with our luciferase findings pSmad2 levels were increased 7 days post stroke (Fig 2G).

In summary, TGF $\beta$ signaling increases 2 fold after $\mathrm{dMCAO}$ stroke, beginning on day 1 and peaking on day 7 , this pattern of increase is preserved in old animals, and absolute TGF $\beta$ signaling in the non-injured brain increases with age. We did not observe a gender difference in these findings or in infarct volume.

\section{TGF- $\beta 1$ is predominantly co-localized with CD68+ cells after stroke}

To determine which cell type is the likely source of increased TGF- $\beta 1$ production after stroke, brain sections were stained with an anti-TGF- $\beta 1$ antibody, colocalized with markers for astrocytes (GFAP), neurons (Millimark Pan-Neuronal marker), and monocytic immune cells (CD68). Although TGF- $\beta 1$ colocalized with all three cell types it was predominantly co-localized with CD68, which marks both activated microglia and macrophages. These cells were located in both the ischemic penumbra and the stroke core. These data suggest that endogenous microglia and invading macrophages are the predominant cell types that make TGF- $\beta 1$ after ischemic brain injury (Fig 3 ).

\section{TGF $\beta$ signaling in neurons, oligodendrocytes and endothelial cells is unchanged by stroke}

To determine which cells are responding to TGF $\beta$ in the uninjured brain and after stroke, brain sections from mice sacrificed at days 1, 3, 7, 14 and 21 after stroke and at 21 days after sham surgery were double-labeled with anti-pSmad2, a marker of TGF $\beta$ signaling, and markers of neurons (Millimark Pan Neuronal marker), oligodendrocytes (CAII), endothelial cells ( $\beta$-dystroglycan), astrocytes (GFAP) and activated microglia and macrophages (CD68). We found that neurons uniformly respond to TGF $\beta$ in the absence of injury and at all time points after stroke, with $100 \%$ of neurons co-localizing with pSmad2 (Fig 4A and Fig 5A; see additional file 1 to confirm colocalization by 3D reconstruction). We also found that the majority (approximately 70\%) of oligodendrocytes consistently respond to TGF $\beta$ in the absence of injury and did not observe any differences after stroke (Fig 4B and Fig 5B; see additional file 2 to confirm colocalization by $3 \mathrm{D}$ reconstruction). There was no qualitative difference in the intensity of colocalization of pSmad2 with neurons or oliogdendrocytes, at any time point after stroke. This data suggests that the response of neurons and oligodendrocytes to TGF $\beta$ is unchanged by ischemic injury. Unlike neurons and oligodendrocytes, $\beta$-dystroglycan rarely colocalized with pSmad2 in the absence of injury and after stroke, indicating that endothelial cells in adult brain seldom signal via $\mathrm{pSmad} 2$ in response to TGF $\beta$ (Fig $4 \mathrm{C}$; see additional file 3 to confirm colocalization by $3 \mathrm{D}$ reconstruction). This data was consistent across age and gender.

\section{TGF $\beta$ signaling increases in astrocytes and microglia/ macrophages after stroke}

Although we found no difference in TGF $\beta$ signaling in neurons, oligodendrocytes and endothelial cells after stroke, we did find a qualitative increase in TGF $\beta$ signaling in astrocytes and microglia/macrophages. In the days following stroke, pSmad2 immunoflorescence became brighter in the border of the lesion (Fig 6A) compared to sham mice that showed even pSmad2 immunoflorescence throughout the cortex (Fig 6B). Concurrently, GFAP and CD68 immunoreactivity increased in the same location and the GFAP and CD68 positive cells co-localized intensely with pSmad2 (Fig 7 \&8; see additional files 4 and 5 to confirm colocalization by $3 \mathrm{D}$ reconstructions). This was the case in young and old mice of each gender and increased co-localization of GFAP and CD68 with pSmad2 corresponded with the profile of increased TGF $\beta$ signaling after stroke we observed by real time imaging (Fig 2C-F). These data suggest that increased TGF $\beta$ signaling in the brain after stroke is due to responses from lesional and perilesional astrocytes and monocytic lineage immune cells.

\section{Discussion}

We show here for the first time that TGF $\beta$ signaling in the brain increases in the first week after stroke in both young and old mice. We incorporated 18 month old mice into this study because nearly three quarters of all strokes occur in people over the age of 65 and there is a large knowledge gap regarding how mechanisms of recovery change with age. Similarly, we performed this study on mice of both genders in order to determine if there is a sex difference in TGF $\beta$ signaling after stroke. In 5 month old mice, TGF $\beta$ signaling increased 2 fold over baseline in the first week after stroke, before beginning to return to pre-stroke levels. This profile of 

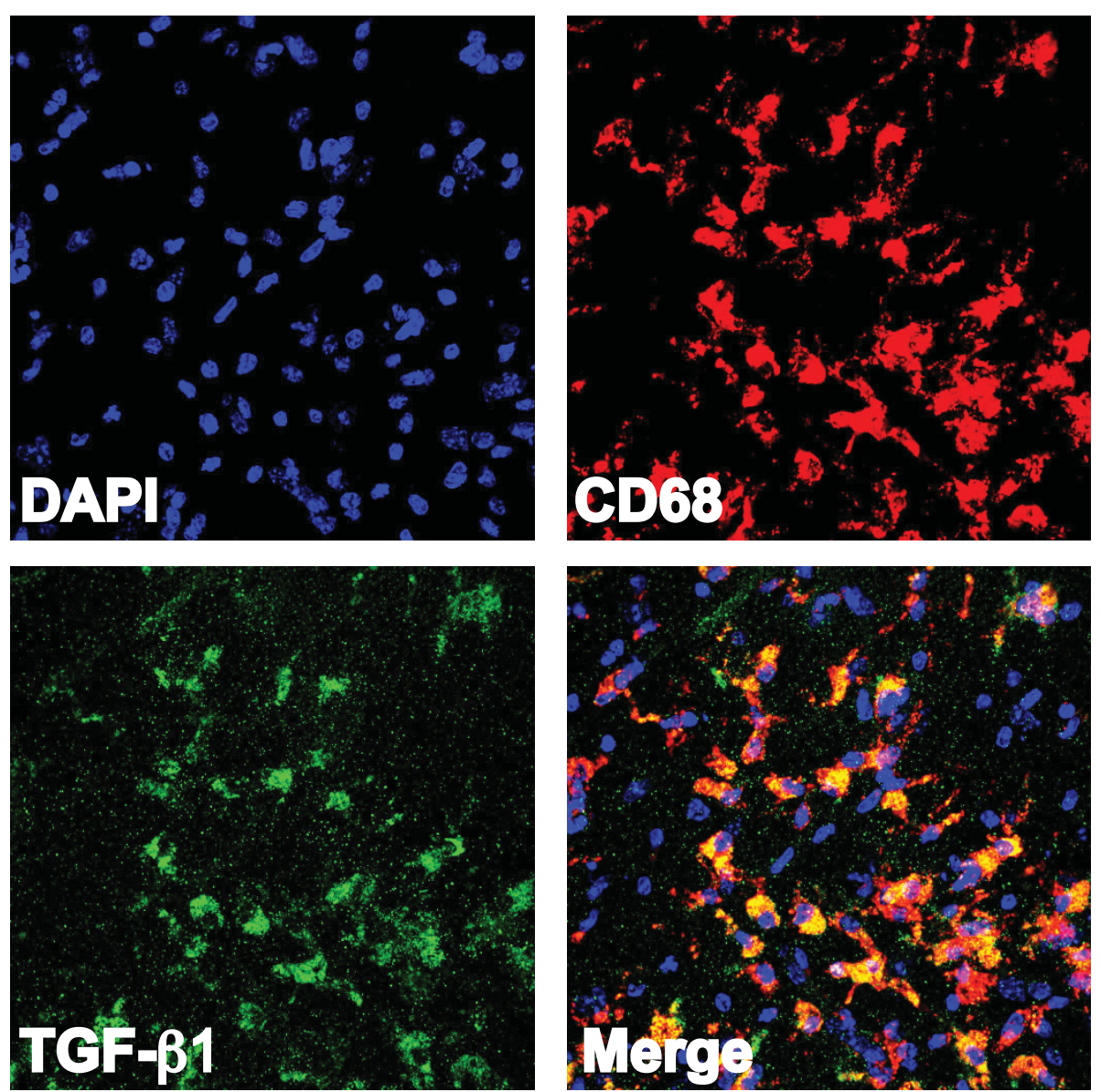

Figure 3 TGF- $\beta 1$ is predominantly colocalized with CD68+ cells after stroke. Representative image (40X) showing CD68 (activated microglia/macrophages) co-localized with TGF- $\beta 1$ after stroke (images are from a young female mouse at day 3 post stroke.

increased TGF $\beta$ signaling was similar in 18 month old mice, although the absolute level of TGF $\beta$ signaling was significantly higher in the older animals. This could be in response to greater damage in the older animals as we found that lesion size was over double the volume compared to the younger mice. Baseline TGF $\beta$ signaling was also higher in the aged animals and so increased TGF $\beta$ signaling after stroke may also be a reflection of their higher baseline level. Our finding that infarct volume is increased in older animals conflicts with other studies that report that infarct volume is not increased in older subjects $[18,20]$. However, these studies used the suture model of stroke, which causes a larger lesion than the dMCAO model of stroke used here. Studies that use a similar cortical model of stroke to the dMCAO model find that lesion size is increased in aged rats relative to young [19]. Therefore the impact of age on lesion size appears to be stroke model dependent.

Every cell type in the brain has been shown to be capable of making TGF- $\beta 1$ and increases in TGF- $\beta 1$ mRNA have been demonstrated after stroke $[1,21]$. To discover which cell type is predominantly responsible for post stroke production of TGF- $\beta 1$ we co-localized an antiTGF- $\beta 1$ antibody with markers of different brain cell types. We found that TGF- $\beta 1$ predominantly colocalized with CD68, a marker of activated microglia and macrophages, indicating that they are likely to be the cells that produce it after stroke.

While there is ample evidence that TGF $\beta$ production is increased after stroke, as it is after many kinds of brain injury, it was not known whether increased TGF $\beta$ production translates to an increased response, because all TGF $\beta$ isoforms are excreted as an inactive form that lies inert in the extracellular matrix [22]. TGF $\beta$ receptor insertion into the membrane is highly regulated and the intracellular Smad pathways that transmit TGF $\beta$ signals interact with a complex array of other kinase substrates [23]. Therefore, although many of the proteins that activate TGF $\beta$ s are upregulated after brain injury, such as reactive oxygen species, metalloproteases, plasmin, and thrombospondin [22], it was not known if increases in TGF- $\beta 1$ mRNA would correlate directly with increased 


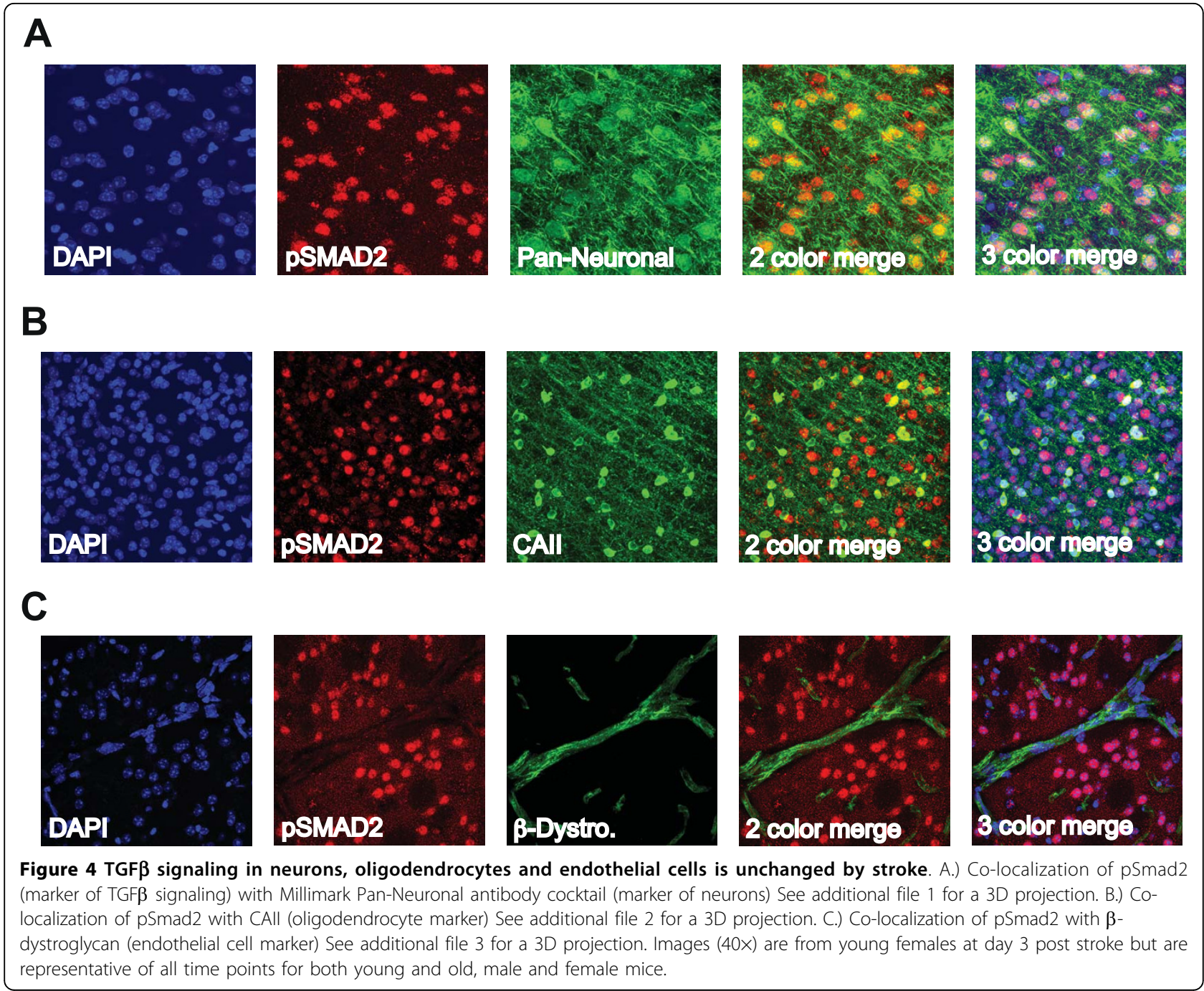

TGF $\beta$ signaling after stroke. And in fact we found that TGF $\beta$ signaling was regulated differently in different cell types after stroke.

TGF- $\beta 1$ is strongly neuroprotective, at least in part due to direct effects in neurons [11,24-28]. It is also involved in synapse formation, the balance of excitatory and inhibitory transmission in the hippocampus and plasticity in multiple circuits [29]. We found that TGF $\beta$ signaling in neurons is widespread and consistent before stroke and that there are no obvious quantitative or qualitative differences after stroke. This suggests that while TGF $\beta$ signaling plays an important constitutive function in neurons, the physiological role of increased TGF- $\beta 1$ after stroke may not be to signal to neurons.

Surprisingly, we also found that the majority of oligodendrocytes in the brain are responding to TGF $\beta$ in the absence of injury and after stroke. This suggests that TGF $\beta$ signaling plays a physiological role in oligodendrocytes that was heretofore unappreciated. As with neurons, co-localization of pSmad2 with oligodendrocytes did not increase after stroke suggesting that the role of increased TGF- $\beta 1$ after stroke is not to communicate to this cell type. Whether constitutive TGF $\beta$ signaling in oligodendrocytes is important for their function or survival remains to be elucidated.

We rarely found co-localization of pSmad2 with endothelial cells. Signal transduction by TGF $\beta$ family members is mediated via specific heteromeric complexes of type I and type II serine/threonine kinase receptors. In most cells TGF $\beta$ signals via the type I receptor ALK5 but in endothelial cells it can also signal via the type I receptor ALK1 [7,30]. ALK5 induces phosphorylation of Smads 2 and 3 and ALK1 mediates phosphorylation of Smads 1, 5 and 8. Endothelial cells may not co-localize with pSmad2 due to the activation of the ALK1 pathway. Thus endothelial cells may still respond to TGF $\beta$ in the brain at baseline and after stroke but by activation of the Smads downstream of ALK1. 


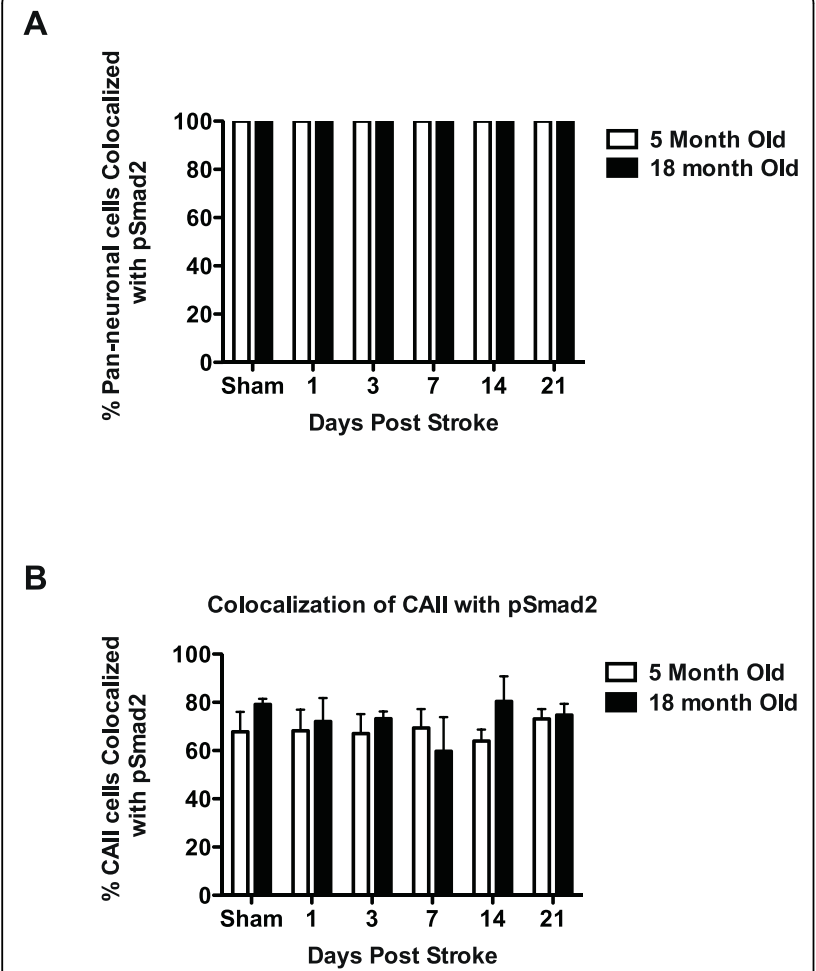

Figure 5 Co-localization of pSmad2 with neurons and oligodendrocytes after stroke. A.) Both before and after stroke $100 \%$ of neurons in the cortex are co-localized with pSmad2 in young and old mice. B.) Both before and after stroke $70 \%$ of oligodendrocytes in the cortex are co-localized with pSmad2 in young and old mice.
Our data demonstrates that CD68+ activated microglia and macrophages respond in an autocrine manner to the TGF- $\beta 1$ they produce. We found that co-localization of CD68 with pSmad2 increased in parallel with TGF $\beta$ signaling reporter gene expression after stroke. TGF $\beta$ can exert either anti-inflammatory or pro-inflammatory effects in a context-dependent fashion. However, in the majority of contexts TGF- $\beta 1$ appears to play an anti-inflammatory role and moderate the production of neurotoxic pro-inflammatory cytokines. It drives the differentiation of regulatory $\mathrm{T}$ cells and M2c macrophages that resolve immune responses, and resolves the inflammatory process in myocardial ischemia $[7,31,32]$. A function of TGF- $\beta 1$ after stroke may be to drive the differentiation of monocytic lineage cells - both activated microglia and macrophages - into an M2c phenotype to enable a similar wound healing response to that identified in myocardial ischemia.

Our data also demonstrates that co-localization of pSmad2 with activated astrocytes increases after stroke. There is strong in vitro and in vivo evidence that TGF $\beta$ plays an important role in generating reactive astrogliosis, and that reactive astrocytes participate in innate immune responses. Overexpression of TGF $\beta$ chronically leads to reactive astrogliosis, and these mice display exacerbated reactive astrogliosis in a stab wound model [33]. Injection of TGF- $\beta 1$ also increases reactive astrogliosis after stab wound [34], while injection of neutralizing antibodies inhibits expression of fibrogenic proteins, including
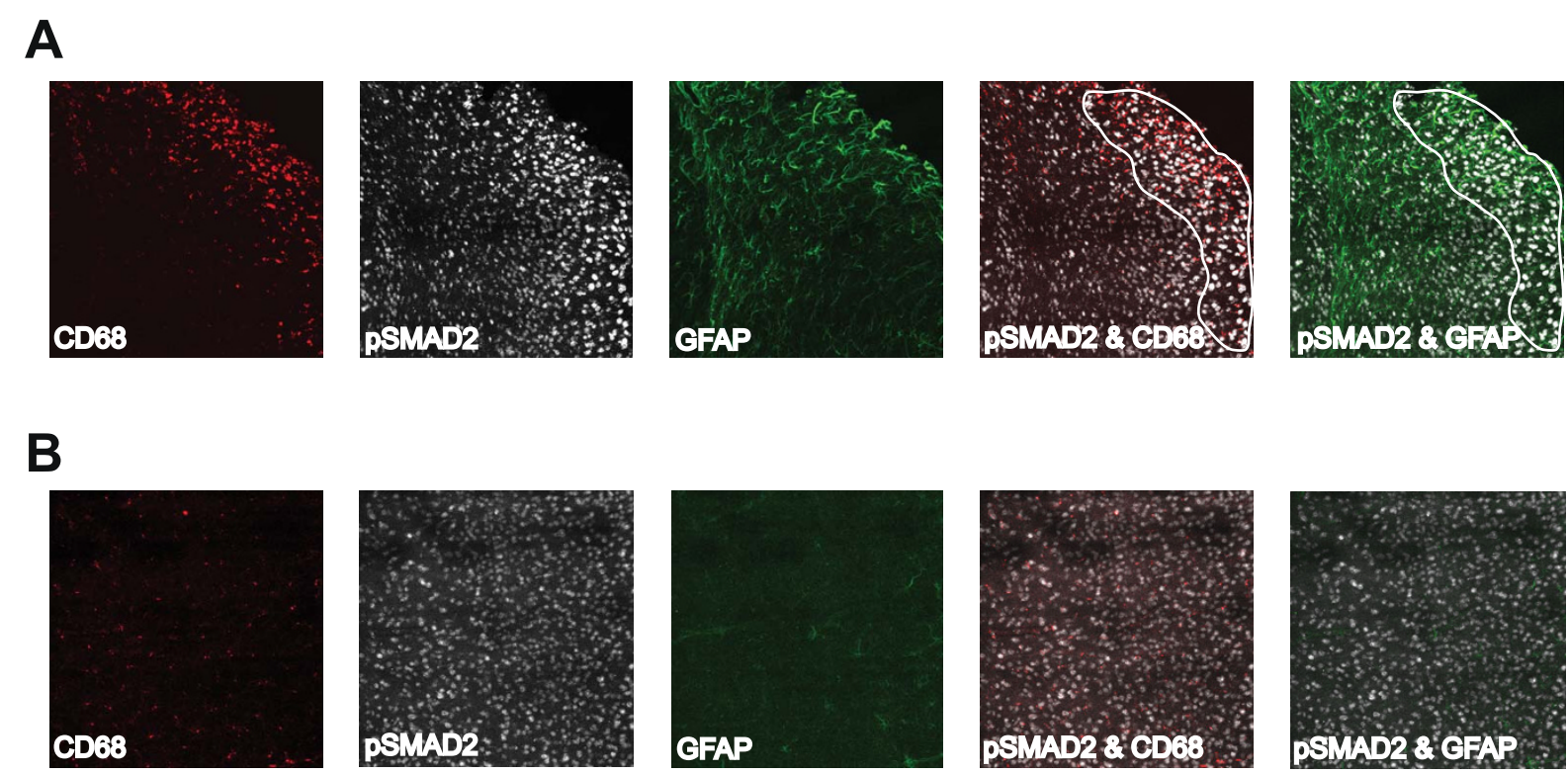

Figure 6 pSmad2 immunoreactivity is increased in the stroke penumbra and colocalizes with CD68 and GFAP. Representative images (10X) showing CD68 and GFAP colocalized with pSmad2 after stroke (image A is from a young female mouse at day 14 post stroke, image B is from a young female mouse after sham surgery). There is a qualitative increase in pSmad2 immunoflorescence in the infarcted region (outlined) with the brightest cells co-localizing with CD68 and GFAP. 


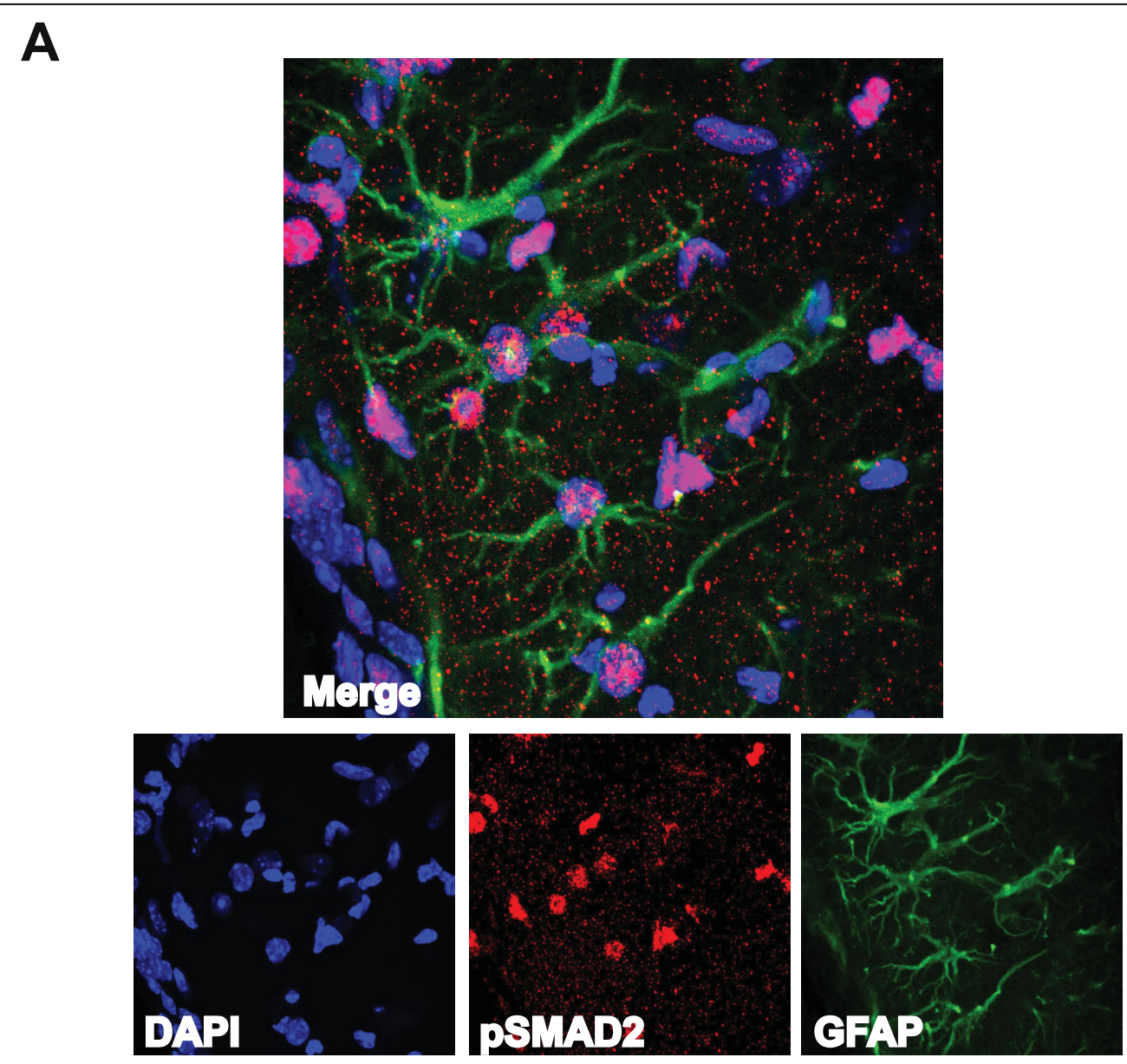

B

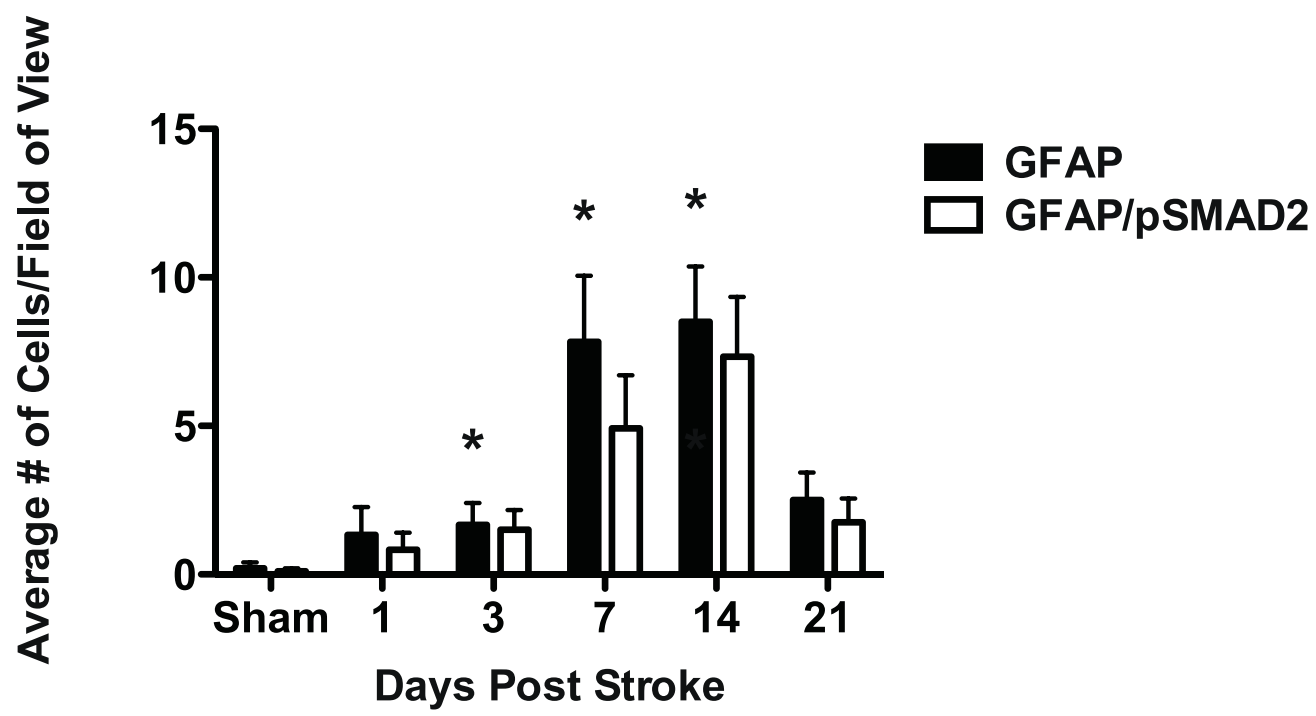

Figure 7 TGF $\beta$ signaling in reactive astrocytes increases in the stroke penumbra after stroke. A.) Representative image (40x) showing GFAP (astrocytes) colocalized with pSmad2 after stroke. Image is from a young female mouse at day 14 post stroke. See additional file 4 for a 3D projection B.) GFAP immunoreactivity increases in the first 7 days after stroke with the majority of astrocytes colocalizing with $\mathrm{pSmad} 2\left(^{*} \mathrm{p}<\right.$ 0.05 compared to sham). 


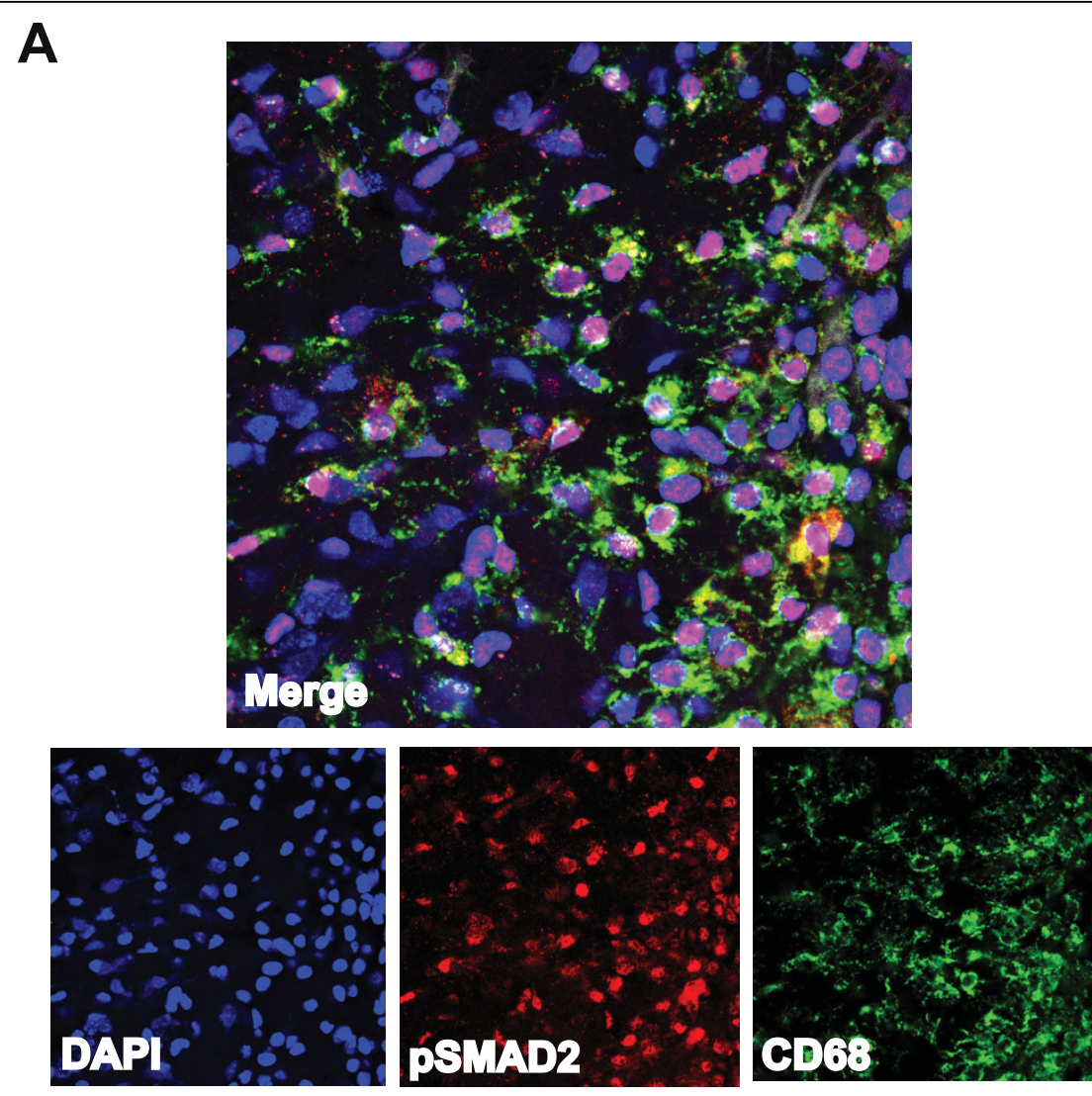

B
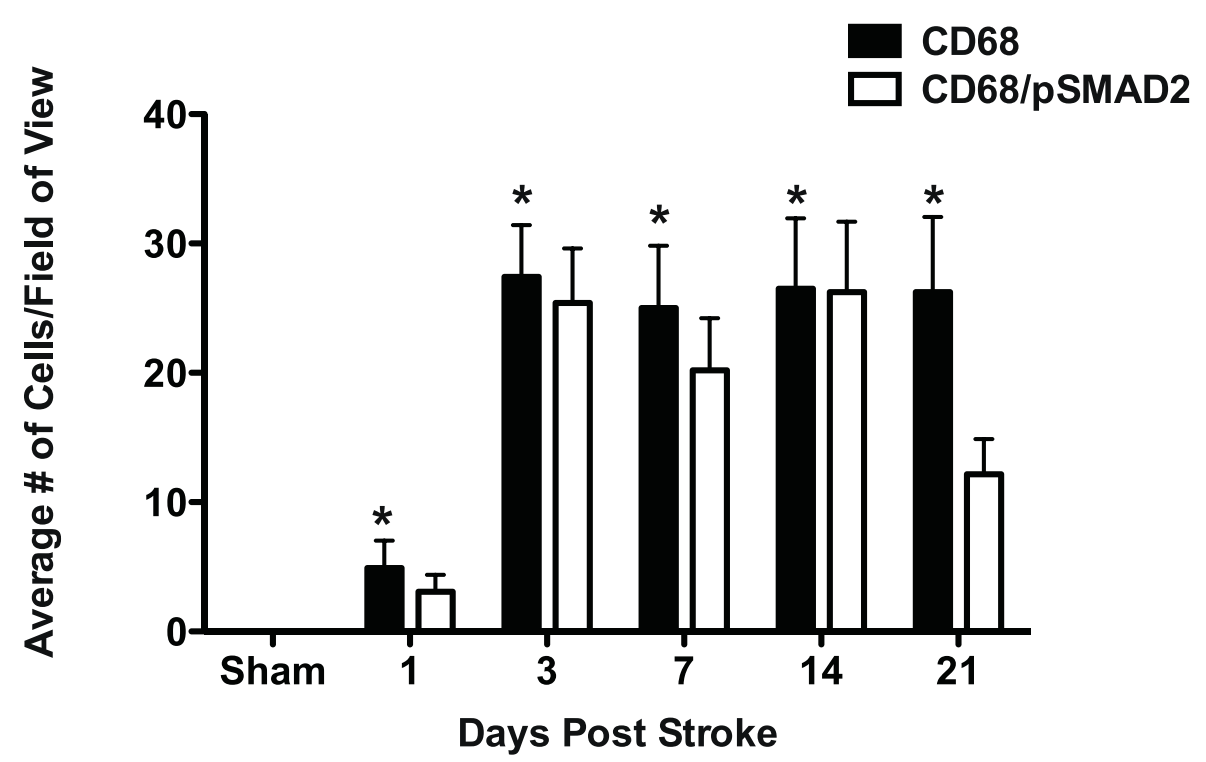

Figure 8 TGF $\beta$ signaling in activated microglia/macrophages increases in the stroke penumbra after stroke. A.) Representative image (40X) showing CD68+ activated microglia and macrophages) co-localized with pSmad2 after stroke. Image is from a young female mouse at day 3 post stroke. See additional file 5 for a 3D projection. B.) CD68 immunoreactivity increases in the first 3 days after stroke with the majority of CD68+ cells co-localizing with pSmad2 (*p $<0.05$ compared to sham). 
proteoglycans, and reduces reactive astrogliosis [5,34]. Finally, Smad3 knockout mice display faster wound healing and decreased scar formation after brain stab injury [6]. Prior to this study no investigation addressed whether it is TGF $\beta$ signaling in astrocytes that leads to reactive astrogliosis, but we show here that astrocytes do respond to post stroke increases in TGF $\beta$. Together this supports the hypothesis that TGF $\beta$ signaling in astrocytes directly mediates astrogliosis after stroke. Astrogliosis may have a detrimental effect on recovery due to astrocytes extending processes in the infarct border to encompass the developing infarct, thereby inhibiting neuronal plasticity and the formation of new axons and blood vessels in the infarcted region [35].

As the brain ages it becomes characterized by a low grade chronic pro-inflammatory state and responds with an earlier and stronger innate immune response following stroke [20]. We found that there is increased TGF $\beta$ signaling in the aged brain in the absence of injury and this may be part of an anti-inflammatory strategy employed by the aging brain to counter the primed proinflammatory state. Our findings are consistent with others who have shown that in aging the expression of TGF $\beta$ receptors increases, as does the expression of TGF- $\beta 1[2,20,36,37]$. Conversely, since the data we present here strongly suggests that TGF $\beta$ signaling is important for neuronal function, increased TGF $\beta$ signaling in the aged brain may be required to maintain normal neuronal function in the face of age related changes. More research is needed to determine which of these scenarios is correct.

\section{Conclusions}

These results show that TGF $\beta$ signaling in the brain does not oscillate with the estrus cycle and that it increases 2 fold after the dMCAO model of stroke in young and old animals of each gender. Astrocytes and CD68+ innate immune cells are the cell types that respond to increased levels of TGF $\beta$ after stroke and they do so during the first week after stroke, coincident with glial scar formation. Given TGF $\beta$ s effects on monocytic lineage cells and astrocytes we hypothesize that the role of increased TGF $\beta$ signaling after stroke is to regulate glial scar formation and the polarization of invading innate immune cells and resident microglia.

\section{Additional material}

Additional file 1: 3D projection of neurons colocalizing with pSmad2. 3D projection of Figure 4A.

Additional file 2: 3D projection of oligodendrocytes colocalizing with pSmad2. 3D projection of Figure 4B.

Additional file 3: 3D projection of lack of colocalization of endothelial cells with pSmad2. 3D projection of Figure 4C.
Additional file 4: 3D projection of astrocytes colocalizing with pSmad2. 3D projection of Figure 7A.

Additional file 5: 3D projection of microglia colocalizing with pSmad2. 3D projection of Figure 8A.

\section{Acknowledgements}

We thank Jullet Han and Geoffrey Stanley for valuable technical assistance and Dr Tony Wyss-Coray for providing us with SBE-LuCRT mice. This work was supported by an Ellison Medical Foundation/AFAR Postdoctoral Fellowship to KPD and KO8 NS050304-01A2 to MSB.

\section{Authors' contributions}

KPD performed the surgery, estrus determination and live imaging. KPD, EC and LEM carried out the immunoflorescence. KPD and MSB conceived of the study, and participated in its design and coordination and helped to draft the manuscript. All authors read and approved the final manuscript.

\section{Competing interests}

The authors declare that they have no competing interests.

Received: 29 July 2010 Accepted: 11 October 2010

Published: 11 October 2010

\section{References}

1. Buckwalter M, Wyss-Coray $T$ : Modelling neuroinflammatory phenotypes in vivo. J Neuroinflammation 2004, 1:10.

2. Finch CE, Laping NJ, Morgan TE, Nichols NR, Pasinetti GM: TGF-b1 is an organizer of responses to neurodegeneration. J Cell Biochem 1993, 53:314-322.

3. Unsicker K, Flanders KC, Cissel DS, Lafyatis R, Sporn MB: Transforming growth factor beta isoforms in the adult rat central and peripheral nervous system. Neuroscience 1991, 44:613-625.

4. Buisson A, Lesne S, Docagne F, Ali C, Nicole O, Mackenzie ET, Vivien D: Transforming growth factor-beta and ischemic brain injury. Cell $\mathrm{Mol}$ Neurobiol 2003, 23:539-550.

5. Moon LD, Fawcett JW: Reduction in CNS scar formation without concomitant increase in axon regeneration following treatment of adult rat brain with a combination of antibodies to TGFbeta1 and beta2. Eur J Neurosci 2001, 14:1667-1677.

6. Wang $Y$, Moges $H$, Bharucha $Y$, Symes A: Smad3 null mice display more rapid wound closure and reduced scar formation after a stab wound to the cerebral cortex. Exp Neurol 2007, 203:168-184.

7. Bujak M, Frangogiannis NG: The role of TGF-beta signaling in myocardial infarction and cardiac remodeling. Cardiovasc Res 2007, 74:184-195.

8. Yamashita K, Gerken U, Vogel P, Hossmann K, Wiessner C: Biphasic expression of TGF-beta1 mRNA in the rat brain following permanent occlusion of the middle cerebral artery. Brain Res 1999, 836:139-145.

9. Wang X, Yue TL, White RF, Barone FC, Feuerstein GZ: Transforming growth factor-beta 1 exhibits delayed gene expression following focal cerebral ischemia. Brain Res Bull 1995, 36:607-609.

10. Ma M, Ma Y, Yi X, Guo R, Zhu W, Fan X, Xu G, Frey WH, Liu X: Intranasal delivery of transforming growth factor-beta1 in mice after stroke reduces infarct volume and increases neurogenesis in the subventricular zone. BMC Neurosci 2008, 9:117.

11. Ruocco A, Nicole O, Docagne F, Ali C, Chazalviel L, Komesli S, Yablonsky F, Roussel S, MacKenzie ET, Vivien D, Buisson A: A transforming growth factor-b antagonist unmasks the neuroprotective role of this endogenous cytokine in excitotoxic and ischemic brain injury. J Cereb Blood Flow Metab 1999, 19:1345-1353.

12. Kannel WB: The demographics of claudication and the aging of the American population. Vasc Med 1996, 1:60-64

13. Luo J, Ho PP, Buckwalter MS, Hsu T, Lee LY, Zhang H, Kim DK, Kim SJ, Gambhir SS, Steinman L, Wyss-Coray T: Glia-dependent TGF-beta signaling, acting independently of the $\mathrm{TH} 17$ pathway, is critical for initiation of murine autoimmune encephalomyelitis. J Clin Invest 2007 117:3306-3315

14. Tamura A, Graham DI, McCulloch J, Teasdale GM: Focal cerebral ischaemia in the rat: 1 . Description of technique and early neuropathological 
consequences following middle cerebral artery occlusion. J Cereb Blood Flow Metab 1981, 1:53-60.

15. Massagué J, Wotton D: Transcriptional control by the TGF-b/Smad signaling system. EMBO J 2000, 19:1745-1754.

16. Shi Y, Massague J: Mechanisms of TGF-beta signaling from cell membrane to the nucleus. Cell 2003, 113:685-700.

17. Lin AH, Luo J, Mondshein LH, Ten Dijke P, Vivien D, Contag CH, WyssCoray T: Global Analysis of Smad2/3-Dependent TGF-\{beta\} Signaling in Living Mice Reveals Prominent Tissue-Specific Responses to Injury. J Immunol 2005, 175:547-554.

18. Badan I, Buchhold B, Hamm A, Gratz M, Walker LC, Platt D, Kessler C, PopaWagner A: Accelerated glial reactivity to stroke in aged rats correlates with reduced functional recovery. I Cereb Blood Flow Metab 2003, 23:845-854.

19. Kharlamov A, Kharlamov E, Armstrong DM: Age-dependent increase in infarct volume following photochemically induced cerebral infarction: putative role of astroglia. J Gerontol A Biol Sci Med Sci 2000, 55:B135-141, discussion B142-133.

20. Petcu EB, Sfredel V, Platt D, Herndon JG, Kessler C, Popa-Wagner A: Cellular and molecular events underlying the dysregulated response of the aged brain to stroke: a mini-review. Gerontology 2008, 54:6-17.

21. Krupinski J, Kumar P, Kumar S, Kaluza J: Increased expression of TGF-b1 in brain tissue after ischemic stroke in humans. Stroke 1996, 27: 852-857.

22. Noble NA, Harper JR, Border WA: In vivo interactions of TGF-beta and extracellular matrix. Prog Growth Factor Res 1992, 4:369-382.

23. Ten Dijke P, Hill CS: New insights into TGF-beta-Smad signalling. Trends in Biochemical Sciences 2004, 29:265-273.

24. Pang L, Ye W, Che XM, Roessler BJ, Betz AL, Yang GY: Reduction of inflammatory response in the mouse brain with adenoviral-mediated transforming growth factor-b1 expression. Stroke 2001, 32:544-552.

25. Buisson A, Nicole O, Docagne F, Sartelet H, Mackenzie ET, Vivien D: Upregulation of a serine protease inhibitor in astrocytes mediates the neuroprotective activity of transforming growth factor beta1. FASEB $J$ 1998, 12:1683-1691.

26. Tesseur I, Zou K, Esposito L, Bard F, Berber E, Can JV, Lin AH, Crews L, Tremblay $P$, Mathews $P$, et al: Deficiency in neuronal TGF-beta signaling promotes neurodegeneration and Alzheimer's pathology. I Clin Invest 2006, 116:3060-3069.

27. Tesseur I, Wyss-Coray T: A role for TGF-beta signaling in neurodegeneration: evidence from genetically engineered models. Curr Alzheimer Res 2006, 3:505-513.

28. Knoferle J, Ramljak S, Koch JC, Tonges L, Asif AR, Michel U, Wouters FS, Heermann $S$, Krieglstein $K$, Zerr I, et al: TGF-beta 1 enhances neurite outgrowth via regulation of proteasome function and EFABP. Neurobiol Dis 2010, 38:395-404.

29. Sun M, Gewirtz JC, Bofenkamp L, Wickham RJ, Ge H, O'Connor MB: Canonical TGF-beta signaling is required for the balance of excitatory/ inhibitory transmission within the hippocampus and prepulse inhibition of acoustic startle. J Neurosci 2010, 30:6025-6035.

30. Goumans MJ, Valdimarsdottir G, Itoh S, Rosendahl A, Sideras P, ten Dijke P: Balancing the activation state of the endothelium via two distinct TGF-b type I receptors. EMBO J 2002, 21:1743-1753.

31. Serhan CN, Savill J: Resolution of inflammation: the beginning programs the end. Nat Immunol 2005, 6:1191-1197.

32. Mantovani A, Sica A, Sozzani S, Allavena P, Vecchi A, Locati M: The chemokine system in diverse forms of macrophage activation and polarization. Trends Immunol 2004, 25:677-686.

33. Wyss-Coray T, Borrow P, Brooker MJ, Mucke L: Astroglial overproduction of TGF-b1 enhances inflammatory central nervous system disease in transgenic mice. J Neuroimmunol 1997, 77:45-50.

34. Logan A, Berry M, Gonzalez A, Frautschy S, Sporn M, Baird A: Effects of transforming growth factor beta 1 on scar production in the injured central nervous systems of the rat. Eur J Neurosci 1994, 6:355-363.

35. Liu BP, Cafferty WB, Budel SO, Strittmatter SM: Extracellular regulators of axonal growth in the adult central nervous system. Philos Trans $R$ Soc Lond B Biol Sci 2006, 361:1593-1610.

36. Bye N, Zieba M, Wreford NG, Nichols NR: Resistance of the dentate gyrus to induced apoptosis during ageing is associated with increases in transforming growth factor-b1 messenger RNA. Neuroscience 2001, 105:853-862.
37. Nichols NR: Glial responses to steroids as markers of brain aging. J Neurobiol 1999, 40:585-601.

doi:10.1186/1742-2094-7-62

Cite this article as: Doyle et al:: TGF $\beta \beta$ signaling in the brain increases with aging and signals to astrocytes and innate immune cells in the weeks after stroke. Journal of Neuroinflammation 2010 7:62.

\section{Submit your next manuscript to BioMed Central and take full advantage of:}

- Convenient online submission

- Thorough peer review

- No space constraints or color figure charges

- Immediate publication on acceptance

- Inclusion in PubMed, CAS, Scopus and Google Scholar

- Research which is freely available for redistribution

Submit your manuscript at www.biomedcentral.com/submit
C Biomed Central 